\title{
Late-occurring left atrial appendage thrombus after ligation using LARIAT
}

\author{
Alexander G. Truesdell • Chinmay P. Patel • \\ Brijeshwar S. Maini
}

Received: 27 March 2014 / Accepted: 2 May 2014 / Published online: 14 June 2014

(C) Springer Science+Business Media New York 2014

An 83-year-old woman with nonvalvular persistent atrial fibrillation, preserved left ventricular systolic function, prior venous thromboembolism, and recurrent gastrointestinal bleeding preventing long-term anticoagulation underwent successful percutaneous left atrial appendage (LAA) ligation by a combined endocardial and epicardial approach using the LARIAT suture closure device (SentreHeart, Redwood City, CA) (Online Video 1). Immediate post-procedure transesophageal echocardiography (TEE) demonstrated complete LAA sealing (Online Video 2). She was discharged to home without oral anticoagulation due to her bleeding history. Continued surveillance transthoracic echocardiography performed up to 9 months later was unremarkable. Thirteen months postprocedure, she presented to the emergency department complaining of progressive dyspnea. Computed tomography of the chest excluded pulmonary embolism but demonstrated a left atrial filling defect (Fig. 1a). Subsequent TEE confirmed thrombus arising from the ligated LAA protruding into the left atrium (LA) (Fig. $1 \mathrm{~b}$ and Online Video 3). The patient was started on systemic anticoagulation. Future surveillance echocardiography is scheduled at 90 days. This case of late developing LAA thrombus highlights the need for further investigation and longer-term follow-up studies to identify the most appropriate surveillance imaging strategy, assess the risks of recurrent thrombus formation and stroke, and determine
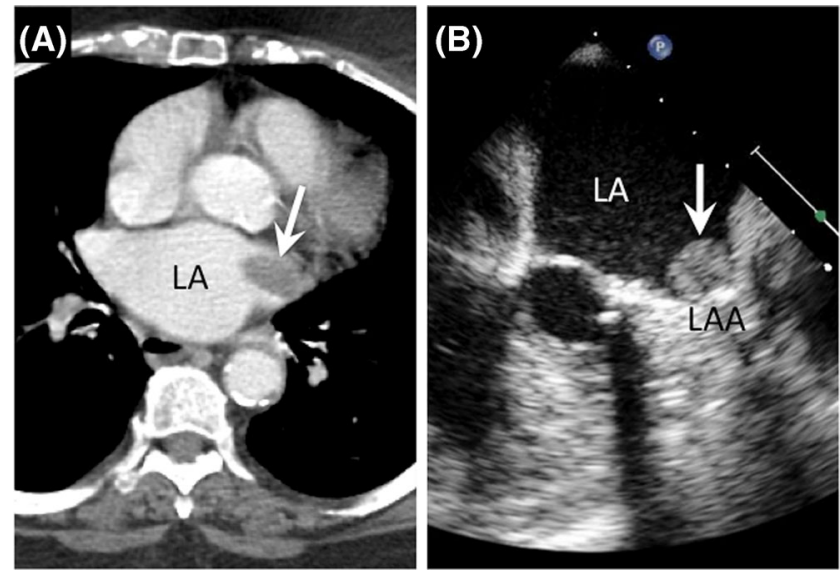

Fig. 1 a Computed tomography of the chest demonstrating a left atrial (LA) filling defect. b Tranesophageal echocardiography confirming thrombus arising from the ligated left atrial appendage protruding into the LA

the need for, and appropriate duration of, systemic anticoagulation following LAA exclusion with the LARIAT device.

Conflict of interest B.S.M. has received honoraria and research grants from Abbott Vascular, Abiomed, Boston Scientific, Infraredx, Medtronic, St. Jude Medical, and Siemens. A.G.T. and C.P.P have no conflicts to declare.
Electronic supplementary material The online version of this article (doi:10.1007/s10840-014-9916-9) contains supplementary material, which is available to authorized users.

A. G. Truesdell $(\bowtie) \cdot$ C. P. Patel $•$ B. S. Maini

PinnacleHealth CardioVascular Institute, Harrisburg, PA, USA

e-mail: atruesdell@pinnaclehealth.org 